\title{
The Perception of Veterinary Students and Faculty on the Use of a Novel Bandage Limb Model for the Purpose of Training
}

\author{
Larry Betance*, Susan Porter, Anne Conan, and Elpida Artemiou
}

Ross University School of Veterinary Medicine, PO Box 334, Basseterre, St Kitts, WI

Received: February 5, 2016 / Accepted: May 25, 2016

\begin{abstract}
In small animal practice, ailments of the extremities are common. Limb bandages are often indicated for degloving injuries, fracture support, and many other orthopedic conditions. Consequently, veterinarians frequently perform bandage placement on their small animal patients. Bandages such as the Modified Robert Jones, Robert Jones, Ehmer sling, and Velpeau sling are some of the commonly applied bandages in practice. Small animal bandaging is an essential component of veterinary skills training. However, many veterinary curricula offer very little if any practical bandage training during the student's pre-clinical veterinary education. At Ross University School of Veterinary Medicine (RUSVM), through collaboration with Veterinary Simulators Industries Ltd ${ }^{a}$ a bandage limb model was developed to address the lack of practical training and to help minimize live animal use for bandage training. In 2014 a questionnaire was conducted with RUSVM faculty and second year veterinary students to determine their perception of the use of the bandage limb model for the purpose of teaching small animal bandaging techniques. Participants included eight faculty who were selected based on their experience with bandaging and 110 students enrolled in the Surgery One Laboratory course. The questionnaire utilized a five-point Likert scale. The median of overall perception scores of the bandage limb model were 33 (out of $\mathbf{4 0}$ ) for students and $\mathbf{4 6 . 5}$ (out of 50 ) for faculty. These results suggest that veterinary students and faculty perceive the bandage limb model as an acceptable alternative that would be useful in teaching veterinary students small animal bandaging techniques. Further studies are required to evaluate the effectiveness of the model in teaching bandaging skills in comparison to the use of a live animal.
\end{abstract}

Key words: Veterinary Medicine, education, simulation, bandaging, small animal, clinical skills

* Corresponding author: lbetance@rossvet.edu.kn

\section{Introduction}

Bandaging is an essential skill for a small animal practitioner and it is expected that the newly graduating veterinarian has acquired the clinical skills necessary to apply bandages properly (Simpson et al., 2001). Bandages and other dressings are frequently used in small animal practice for purposes such as wound debridement, reduction of dead space, hemorrhage control, delivering medication, reducing pain, immobilization and support of a limb (Pavletic, 2010). Adverse outcomes can occur in a patient who is bandaged by a veterinarian whose experience and practice with bandaging skills is limited (Campbell, 2006). Specifically, if a bandage is placed too loose or without aids to hold the bandage material in place, the bandage can slip, thus exposing a wound to the environment. If a bandage is placed too tight, the bandage could compromise circulation to the limb (Pavletic, 2010). Circulatory compromise from a tight bandage can result in surgical debridement, toe amputations, or even total limb amputation. In rare cases, it is reported that tight bandage placement resulted in patient death (Anderson and White, 2000). Prevention of such complications can be achieved through appropriate and repetitious training that is supported with bandage monitoring and feedback (Anderson and White, 2000).

The teaching and learning of bandaging to pre-clinical students includes two vital components; teaching the student appropriate use of various bandaging materials along with instruction on proper bandaging techniques (Donelan, 2003). Often veterinary institutions utilize live animals in the training of bandaging; however, this practice has become controversial (Baver, 1993). As the public's and students' views on animal welfare change, and as budgetary constraints continue to limit resources, there has been an increasing demand for the use of models in veterinary education (Krebsbash, 2011). Additional concerns with the use of live animals include the need for a large supply of animals in order to comply with the policies and standards of the 
Institutional Animal Care and Use committee (IACUC), the need for sedation of the animal subjects, and finally the requirements of increased support staff to provide appropriate monitoring of the sedated patients (Krebsbash, 2011). Another challenge faced in teaching and learning bandaging skills relates to the increase in veterinary class sizes, that unequivocally, adds strain to the already limited available resources when using live animals in a teaching setting (Neubert, 2010; Douglas, 2009).

Many veterinarians report being effectively trained with the use of models (Fletcher et al., 2012 ; Valliyate et al., 2012). Meta-analysis of studies comparing the utility of simulation-based medical education with more didactic methods showed a significant improvement in the development of clinical skills in students trained using immersive simulation techniques (Fletcher et al., 2012. Determining content evidence based on expert evaluation of a training model is one method for ensuring that the educational process is appropriate and useful for the students (Downing, 2011 ; Cook et al., 2014). Recently validated veterinary models include a dental simulation model which has been effective in training students to perform a professional dental cleaning and operate a dental machine (Lumbis et al., 2012). An equine joint injection model was also evaluated with results indicating that comfort levels in this skill increased through the practice and immediate feedback provided by the model (Fox et al., 2013). These models reflect the current trend in veterinary education which is to decrease the number of live animals used for teaching purposes and to replace them with acceptable alternatives (Valliyate et al., 2012).

A bandage limb model would allow students to reinforce their learned skills independently and in small group practice (Valliyate et al., 2012). Having the limb model available to the students would allow repetitive practice in a safe environment without risk to themselves or to the patient. This would help to improve their confidence in bandaging, and would also improve the student's bandaging skills on a live animal patient (Valliyate et al., 2012). A number of canine limb models are being used for bandage laboratories with some success. Utilization of a canine limb model which could flex and extend with similar resistance and range of motion as a limb of a live canine patient would support the learning of bandaging skills.

The goals of this study were to determine if veterinary students and faculty perceive the bandage limb model as an appropriate model that could be utilized to help students learn small animal bandaging techniques, as well as whether the model could be used as a replacement of the live animal patient for practicing bandage application during laboratory time.

\section{Materials and Methods}

\section{Surgery Laboratory One Course}

Ross University School of Veterinary Medicine (RUSVM) curriculum encompasses three semesters per year (each lasting 15 weeks), with a total of seven preclinical semesters on the island of St. Kitts. After completing the preclinical curriculum, the students attend an affiliated American Veterinary Medical Association (AVMA) accredited veterinary school to perform a year of clinical training (a total of 50 weeks). Six semester students are enrolled in a 2 credit mandatory Surgery Laboratory One course. This course includes a small animal bandaging laboratory along with ten other laboratories that encompass skills in aseptic technique, suturing, fracture repair, as well as skills required for a canine ovariohysterectomy model. At RUSVM, this is the first time in the students' veterinary curriculum that they are exposed to the practical application of small animal bandaging, and bandage placement. The bandage laboratory occurs four times throughout the semester, from weeks 2 through 5 and every sixth semester student must attend one of the four laboratories. Each laboratory has approximately 30 students, divided into groups of 3-4 students. The maximum number of groups per laboratory session is eight. The laboratory is set up with stations around the periphery of a large multipurpose indoor room. Each station is equipped with a stainless steel examination table, along with a Ross University owned clinic dog, and fore/hind limb bandage models. As preparation for the laboratory, students are instructed to review the laboratory syllabus and a power point presentation on small animal bandaging which is available on the RUSVM eCollege educational website ${ }^{b}$. The syllabus contains information pertaining to appropriate laboratory attire, equipment provided for the laboratory, equipment the student should bring to the laboratory, required reading material, and laboratory objectives.

The laboratory commences with the instructor providing a brief introduction of the type of bandages that will be demonstrated and practiced during the laboratory. The students are given instructions to provide sedation to the live animal patients with Acepromazine at a dose of $0.05-0.1 \mathrm{mg} / \mathrm{kg}$ intramuscularly (all doses are confirmed with the instructor prior to administration). This medication is administered in order to facilitate bandage placement for the pre-clinical student. While the medication is taking affect, the instructor provides an informational session that addresses the various bandaging materials used during the laboratory, specific indications for canine and feline limb bandages, and potential complications associated with bandaging. The bandages demonstrated by the instructor and practiced by the students are the modified Robert Jones, the Robert Jones bandage, the Ehmer sling and the Velpeau sling. Typically, with group sizes of 3-4 students, not every student receives the opportunity to practice every bandage demonstrated in a laboratory due to the time constraints (Figure 1).

\section{Bandage Limb Model}

Through collaboration with the small animal surgery instructors at RUSVM and Veterinary Simulator Industries LTD a bandage limb model was designed that was deemed suitable for the bandage laboratory setting. The inner structure of the limb is a metallic skeletal structure with hinged joints to provide resistance to simulate a similar resistance encountered from the limbs of a canine patient. When the limb is flexed or extended by a participant, it recoils back to a natural position when released. The outer skin is a hairless black pigmented silicone material which is malleable in order to stretch in multiple directions with motion of the model "joints". The area between the outer skin 


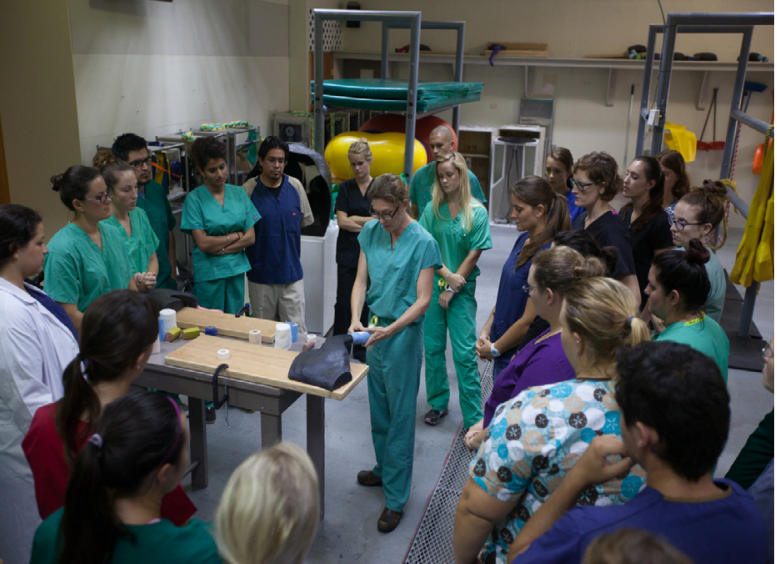

Figure 1. Image of the Bandage Laboratory Instruction.

and the skeleton is filled with soft urethane foam. This foam simulates the soft tissue around the limb in order to maintain normal limb shape. The proximal medial aspects of both the hind and fore limbs have an external threaded female mounting sleeve. This sleeve allows the limb model to be firmly attached to a one-inch thick mounting board through use of a two-inch long 3/8-inch hex head bolt. The area in which the bolt penetrates through the mounting board has been counter sunk to allow the board to rest flat on an examination table. The mounting board is one-foot wide by three feet long. The mounting board is secured to the end of a stainless steel examination table by eight inch "C" clamps placed at each end of the board. This type of attachment prevents the model from sliding during bandage placement. It also allows access to the entire circumference and joints of the model to facilitate bandage placement (Figure 2).

\section{Study Design}

\section{Faculty}

A total of eight small animal clinical faculty members were invited and accepted to participate in the study. These faculty members were selected based on their experience in small animal bandaging given their clinical backgrounds and prac-

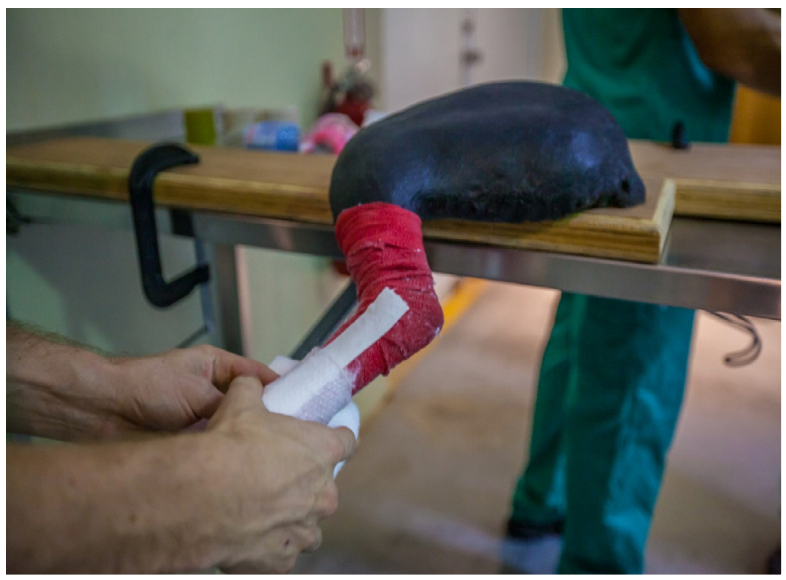

Figure 2. Image of Hind Limb Model. tice. Participating small animal clinical faculty filled out a questionnaire regarding their experience in small animal bandage placement, experience with teaching these skills, as well as their perceived level of experience with small animal bandaging skills. The clinical skills laboratory was specifically set up for the faculty participants who were asked to use the models and apply the same bandages demonstrated in the student laboratories. Upon completion of the bandage laboratory, faculty participants completed a ten-item questionnaire evaluating their perception of the model. Specifically, these questions elicited the faculty's perception in relation to the realism of the model, as well as its usability to teach small animal bandaging techniques to novice veterinary students. Furthermore, the questionnaire included an open ended question for faculty members to provide comments based on their experience with the model. All questions were answered using a five point Likert scale with numeric values of 1 (strongly disagree) to 5 (strongly agree).

\section{Students}

All six semester students participating in the study were enrolled in the Surgery Laboratory One course in the summer semester of 2014. After completing the laboratory, students were asked to complete an eight-item questionnaire. The questions, although slightly different from the faculty's questionnaire, likewise elicited the student's perception of the realism and usability of the model as compared to the use of a live animal for learning small animal bandaging techniques. The same five point Likert scale was used.

The study was approved by the Ross University School of Medicine Institutional Review Board and all participating students and faculty signed an informed consent.

\section{Statistical Analysis}

All analyses were performed using $R$ software ${ }^{c}$. Description of the score distribution (including median, mean, minimum and maximum) was obtained for each question of the faculty and students questionnaires. Analyses were completed separately for faculty and student scores as the administered questions were slightly different. An overall perception score by participant was calculated by summing the score of all questions. The last question of faculty questionnaire ("I have concerns that this model could teach students poor technique") was recoded before summing in order to better correlate the favorable and unfavorable responses with the rest of the questions provided. The overall scores were categorized in 5 categories: Extremely low (faculty scale: 10-17; students scale: $8-14$ ), Low (faculty: 18-25; students: 15-21), Neutral (faculty: 26-34; students: 22 26), High (faculty: 35-42; students: 27-33), and Extremely high (faculty: 43-50; students: 34-40). A factor analysis on the student questionnaire was performed to evaluate internal structure of the questionnaire utilized in the study. The number of factors was determined using the command $n S c r e e$ of the $R$ package nFactors and the model used maximum likelihood estimation to define these factors. 


\section{Results}

Six faculty participants considered themselves as experts, one as proficient and one as novice in bandage application skills. They all had experience performing small animal bandaging on a regular basis prior to and/or during employment at RUSVM. One of the faculty participants doesn't perform bandaging anymore in their current position at RUSVM. Score distribution indicates an overwhelmingly positive response to the bandage model, with medians between 4 and 5 for all the perception questions (Table 1). Five of the faculty participants had an overall score superior to 42 out of 50 (extremely high score), while the 3 others had a score between 35 and 42 (high score). General comments were positive with regards to the model's intended purpose (i.e. "great model", and "good for bandages ") while a few provided recommendations for improving the detail of the model ("providing an articulated hip for internal rotation", or "providing spacing between toes to place cotton padding"). One hundred and ten students volunteered to participate in the bandage model study. Scores for each individual question were high with medians from 3 to 5 (Table 2). The overall score given by the student were classified as high with a mean of 32.8 (Standard Deviation (SD): 3.6) (Figure 3). In reviewing many of the comments provided by the students, they felt the model was "very useful in helping to learn bandaging techniques and that "they really liked the model".

The factor analysis extracted 3 different factors. The first factor encompassed four questions (Q1, Q2, Q5 and Q6) linked with the realism of the model and its usefulness in improving the student confidence and skills (Proportion of variance: $22 \%$ ). The

Table 1. Description of Faculty Perception Scores Regarding the Bandage Limb Model.

\begin{tabular}{|c|c|c|c|}
\hline & Minimum & Median & Maximum \\
\hline 1.The overall size of the model was appropriate for the skill & 4 & 5 & 5 \\
\hline 2.An adequate number of landmark structures were present & 4 & 5 & 5 \\
\hline $\begin{array}{l}\text { 3.The location and feel of the landmark structures was } \\
\text { suitably realistic }\end{array}$ & 4 & 4.5 & 5 \\
\hline 4.The materials looked and felt appropriately realistic & 3 & 4 & 5 \\
\hline 5.The model was easy to use & 4 & 5 & 5 \\
\hline $\begin{array}{l}\text { 6.The model was suitable to teach the preparation and steps } \\
\text { required to perform the skill }\end{array}$ & 4 & 4.5 & 5 \\
\hline $\begin{array}{l}\text { 7. The model was suitable to give students a general idea of } \\
\text { the actual tactile experience when performing this skill }\end{array}$ & 4 & 5 & 5 \\
\hline $\begin{array}{l}\text { 8.I feel that this model would be helpful for students to } \\
\text { practice the skill prior to performing it on a live animal }\end{array}$ & 4 & 4.5 & 5 \\
\hline $\begin{array}{l}\text { 9.I feel that his model is adequate to assess student } \\
\text { performance of the skills stated }\end{array}$ & 4 & 5 & 5 \\
\hline $\begin{array}{l}10.1 \text { have concerns that this model could teach students poor } \\
\text { technique }\end{array}$ & 1 & 1 & 4 \\
\hline
\end{tabular}

Table 2. Description of Student Perception Scores Regarding the Bandage Limb Model.

\begin{tabular}{|c|c|c|c|c|}
\hline & Minimum & Mean (SD) & Median & Maximum \\
\hline $\begin{array}{l}\text { 1.The overall size and shape of the bandage model seemed } \\
\text { suitably realistic for practicing small animal bandage } \\
\text { application }\end{array}$ & 1 & $4.5(0.7)$ & 5 & 5 \\
\hline $\begin{array}{l}\text { 2.The joint resistance on the bandage model seemed } \\
\text { appropriate for application of the Velpeau and Ehmer sling }\end{array}$ & 1 & $3.8(1)$ & 4 & 5 \\
\hline $\begin{array}{l}\text { 3.The use of the bandage model provided a better practical } \\
\text { learning experience than the use of live animals }\end{array}$ & 1 & $2.9(1.1)$ & 3 & 5 \\
\hline $\begin{array}{l}\text { 4.I feel the use of the bandage model can serve as a } \\
\text { replacement for live animals to practice application of the } \\
\text { bandages demonstrated in the laboratory }\end{array}$ & 1 & $2.9(1.1)$ & 3 & 5 \\
\hline $\begin{array}{l}\text { 5.I feel that practicing bandaging on the bandage model can } \\
\text { help increase confidence in applying bandages in the small } \\
\text { animal patient }\end{array}$ & 1 & $4.5(0.7)$ & 5 & 5 \\
\hline $\begin{array}{l}\text { 6.The skills learned using the bandage model should be } \\
\text { valuable when going into general small animal practice }\end{array}$ & 1 & $4.6(0.6)$ & 5 & 5 \\
\hline $\begin{array}{l}\text { 7. When learning a new practical task or skill, the opportunity } \\
\text { to perform the task or skill multiple times enhances the } \\
\text { learning process }\end{array}$ & 4 & $4.9(0.3)$ & 5 & 5 \\
\hline $\begin{array}{l}\text { 8.The ability to perform the bandages demonstrated in } \\
\text { laboratory is an essential day one skill for veterinary } \\
\text { graduates }\end{array}$ & 3 & $4.7(0.5)$ & 5 & 5 \\
\hline
\end{tabular}




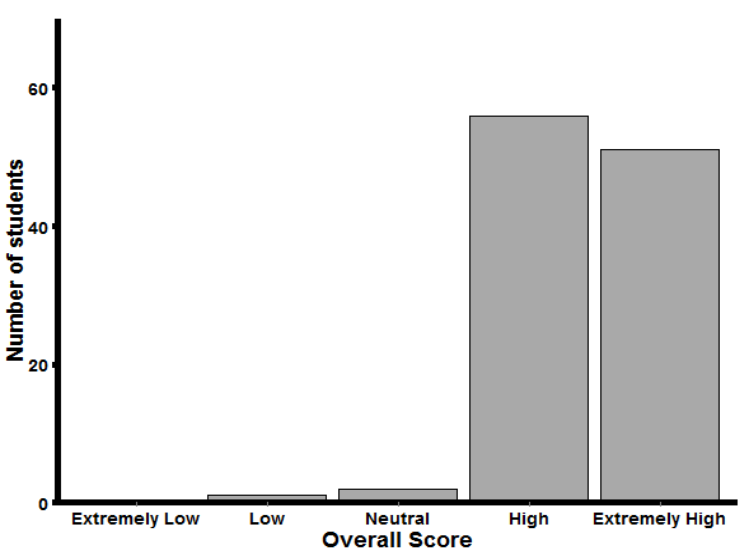

Figure 3. Frequency Distribution of Student Overall Perception Regarding the Bandage Limb Model

second factor (2 questions: Q7 and Q8) described the importance of practicing bandaging multiple times as an essential skill for practice ready graduated veterinarian ( $17 \%$ of variance). The last factor accounted for $13 \%$ of the variance and grouped questions about the model as a replacement for a live patient (Q3 and $Q 4$ ).

\section{Discussion}

The project presented describes the veterinary faculty and student perception of a canine limb model for teaching small animal limb bandaging. In small animal practice, there are a number of different bandages and bandage applications. A small animal practitioner must know the indications, contraindications and applications of the various bandages required by their patients. They must also have the skills required to properly place such bandages (Simpson et al., 2001). Based on the authors' experiences and in speaking with graduates from other AVMA accredited veterinary schools, instruction in the placement of small animal limb bandages is quite limited. Previously at RUSVM, a limited number of students would have just one opportunity to place one type of limb bandage while others in the group observed the bandage placement. Study results support the literature that bandaging is an essential skill for the practice ready veterinarian. One might therefore consider that limited instruction and exposure to limb bandaging would be considered a deficiency in the instruction of necessary skills required for a practice ready small animal veterinarian.

The purpose for developing and evaluating the bandage limb model was to establish a model that could be used in educating and training students in limb bandaging techniques. The Rufus Model ${ }^{\mathrm{d}}$ has been used for this purpose, however this model is large, does not have articulating joints similar to a live patient, and its cost is prohibitive when having to purchase multiple models to accommodate the large class sizes (Fox et al., 2013). A significant benefit to this study, specifically with the use of models within the laboratory, was that each student had the opportunity to place each of the four bandages demonstrated in the laboratory. Previously, due to patient limitations and time considerations, many students had limited opportunities to participate in bandage placement. They spent a majority of the laboratory observing rather than doing and practicing the bandages demonstrated. Other studies have shown that the learning of practical skills is improved when the learner is involved in the case actively as compared to didactic learning (Downing, 2013). This model therefore, which provided simulated front and hind limbs, was a useful learning tool.

The faculty participants that self-identified as experts reported the model would provide an effective tool to educate veterinary students the clinical skill of small animal bandaging. Additionally, the faculty indicated that the model would not teach poor bandaging techniques. Although responses were good in regards to the realism of the model, they were not quite as positive as some of the other faculty responses. Based on comments provided by the faculty, a few deficiencies in the realism of the model were identified. The toes of this model were webbed together. In a real patient, toes are distinctly separated and cotton is often placed between the toes to absorb moisture and to minimize bandage sores. Another deficiency noted by faculty was the inability of the coxofemoral joint to internally rotate. Although the hip joint in our model could flex and extend, the internal rotation is an important aspect to enable limb abduction for proper placement of the Ehmers sling (Tobias and Johnson, 2012). Another limitation of the model was related to the silicone based hairless skin. Application of adhesive tape directly to the skin for the purpose of the "stirrup" placement (used to help hold the bandage in place) was unsuccessful due to poor tape adherence to the model. Following the study, this limitation was minimized by covering the distal portion of the limb with a layer of PetFlexe flexible cohesive bandage. This allowed the adhesive tape to adhere well to the PetFlex ${ }^{e}$ covered model. An alternative approach to consider would be altering the composition or texture to the outer "skin" layer of the model that would be more amendable to stirrup tape application. A further challenge was encountered with the silicone skin. In a live patient, real skin tends to conform well during limb flexion and extension. Although the silicone skin was quite pliable, placement of the non-weight bearing slings occasionally resulted in "bunching" of the elasticized material during extreme flexion. This increased the level of difficulty in appropriately placing the adherent tape for the non-weight bearing slings. Lastly, the model was attached to a large board in order for it to be fixed to an exam table. Having just the limb instead of a limb attached to a body resulted in some conceptual issues when teaching the application of the Velpeau sling. To overcome this difficulty, we secured the forelimb model such that the stabilizing board and thus the limb extended well beyond the end of the exam table. This would allow the student to wrap the Elasticon ${ }^{f}$ tape completely around the board thus simulating a body.

There were many positive aspects of having just the limb model over having an entire mannequin like model to practice bandaging. It would be very difficult to fix and stabilize a full sized mannequin to an examination table. This would likely require the aid of other students to hold the model while one student places a bandage. With our model, students are able to practice and learn bandaging independently without the as- 
sistance from other students. Also, a full sized mannequin is much larger and cumbersome thus creating more issues with moving and storage.

In analyzing the three determined variables provided by the student's perspective regarding the realism of the model in questions $Q 1$ and $Q 2$, the students found that in overall the model was suitably realistic for the bandages practiced. From the questionnaire results and comments the conclusion was reached that the bandage limb model can support the development of student's bandaging skills. In relation to the bandage model as a replacement for live animals, there were more mixed reviews. In analyzing the students' responses from questions Q3 and Q4, it was clear that although the students liked the ability to use the limb model, they did not believe it was a suitable replacement for a live patient. It would only be logical that a veterinarian who will be placing a bandage on a client owned patient would at some point in their training learn to place the bandage on a live patient. However, if they can practice and become more proficient learning this skill on a model it would help minimize some of the pitfalls associated with the use of live animals for teaching. The students believed that the limb model was a useful aid in allowing them to practice bandaging, thus giving them more time to practice without some of the concerns associated with the use of a live patient.

This study received very positive responses from the students regarding the realism of the model and the importance on bandage application in small animal practice. When the students were first exposed to the model, they appeared captivated at the ability of the limb model to flex and extend similar to a live patient. They also seemed surprised at the realistic external appearance of the model and its usability when practicing bandaging. One important aspect was the fact that all students were participating in bandage placement rather than just some. We felt that full student participation along with model realism resulted in the overwhelmingly positive responses from the students.

The questionnaire used for this study was suitable to review the perception of the students toward the model. The factor analysis distinguished clearly three different factors: the realism of the model, the frequency of practicing bandaging and the use of the model as a replacement for a live patient. This questionnaire could be used as a standard in future studies on perception towards bandage limb model.

In conclusion, the development of a bandage limb model that has similar characteristics to the limb of a live canine provides students the opportunity to practice limb bandaging repeatedly and effectively. Although there are limitations to the model, our data strongly support that the model offers sufficient realism to provide an additional effective teaching model during our bandage labs. Validation studies will be the next step in determining the models effectiveness. We acknowledge that further studies must be additionally completed to demonstrate improvements in student's outcomes.

\section{Acknowledgements}

We would like to thank all our participants for supporting our study. Further, we must acknowledge the Center for Innovation in Veterinary and Medical Education, Ross University School of Veterinary Medicine for supporting the study.

\section{Notes}

a: Collaboration between Ross University School of Veterinary Medicine, St Christopher and Nevis (www.rossvet.edu.kn) and Veterinary Simulator Laboratories, Calgary, Alberta, Canada (http://www.vetsimulators.com/).

b: e-College education website. http://www.rossuniversity.net/ c: Rufus bandaging manikin. Rescue critters, 15636 Saticoy street suite A, Van Nuys, Ca 91406.

d: R Core Team R: A language and environment for statistical computing. R Foundation for Statistical Computing, Vienna, Austria 2014 . Available from: http://www.R-project.org/

e: Petflex is a flexible cohesive bandage. Andover Healthcare, Inc. Salisbury, MA 01952

$\mathrm{f}$ : Elasticon is a high twist, cotton elastic cloth tape with rubber based adhesive. Johnson \& Johnson, One Johnson \& Johnson Plaza, New Brunswick, NJ 08933.

\section{References}

Anderson D and RA White (2000) Ischemic bandage injuries: A case series and review of the literature. Veterinary surgery (29): 488498.

Animal use in the DVM program. Tufts University, Cummings School of Veterinary Medicine http://vet.tufts.edu/education/dvm-program/ animal-use/.

Baver MS (1993) A survey of the use of live animals, cadavers, inanimate models, and computers in teaching veterinary surgery. Journal of the american veterinary medical association 203 (7): 1047-1051.

Campbell BG (2006) Dressings, bandages, and splints for wound management in dogs and cats. Veterinary clinics small animal practice (36): 785-786.

Cook DA, B Zendejas, SJ Hamstra, R Hatala, and R Brydges (2014) What counts as validity evidence? Examples and prevalence in a systematic review of simulation-based assessment. Advances in Health Sciences Education 19 (2): 233-250.

Donelan S (2003) Teaching wound care and bandaging: A historical perspective. Wilderness and environmental medicine (14): 47-56.

Douglas J (2009). College of veterinary medicine to increase class size. Virginia Tech news. https://www.vtnews.vt.edu/global_assets/php/ widgets/print-to-pdf/printme.php?url=aHROcDovL3d3dy52dG5Id 3MudnQuZWR 1 L2FydGljbGVzLzlwMDkvMDQvMjAwOSOzMDcua $\mathrm{HRtbA}==\& \mathrm{ct}=\mathrm{vtArticle}$ \&type $=$ print.

Downing SM (2011) Face validity of assessments: faith-based interpretations or evidence-based science? Medical education 40 (8): 7-8.

Fletcher DJ, R Militello, GL Schoeffler, and CL Rogers (2012) Development and evaluation of a high-fidelity canine patient simulator for veterinary clinical training. Journal of veterinary medical education 39 (1): 7-12.

Fox V, C Sinclair, DM Bolt, J Lowe, and R Weller (2013) Design and validation of a simulator for equine joint injections. Journal of vet- 
erinary medical education 40 (2): 152-157.

Krebsbach S (2011) Simulators expand veterinary training and reduce harmful animal use in veterinary schools. Humane society veterinary medical association. http://www.hsvma.org/simulators_expand_training_reduce_harmful_animal_use_veterinary_ schools_042611\#.VrH-zk10xaQ.

Lumbis RH, SP Gregory, and S Baillie (2012) Evaluation of a dental model for training veterinary students. Journal of veterinary medical education 39 (2): 128-135.

Neubert A (2010) Purdue school of veterinary medicine to increase enrollment. Purdue university news service. http://www.purdue.edu/ newsroom/general/2010/100901 SalisburyVet.html.

Pavletic MM (2010) Atlas of small animal wound management. WileyBlackwell, pp. 81-82.

Simpson AM, MA Radlinsky, and BS Beale (2001) Bandaging in dogs and cats: external coaptation. Compendium 23 (2): 157-163.

Tobias KM and SA Johnson (2012) Veterinary small animal surgery. Elsevier, pp. 635.

Valliyate MN, G Robinson, and JR Goodman (2012) Current concepts in simulation and other alternatives for veterinary education: a review. Veterinarni medicina 57 (7): 325-337. 